\title{
Predictive factors affecting cecal intubation failure in colonoscopy trainees
}

Hong-Jun Park', Jin-Heon Hong ${ }^{1}$, Hyun-Soo Kim ${ }^{1,2^{*}}$, Bo-Ra Kim ${ }^{1}$, So-Yeon Park ${ }^{1}$, Ki-Won Jo ${ }^{1}$ and Jae-Woo Kim ${ }^{1}$

\begin{abstract}
Background: Successful cecal intubation (SCI) is not only a quality indicator but also an important marker in a colonoscopy trainee's progress. We conducted this study to determine factors predicting $\mathrm{SCl}$ in colonoscopy trainees, and to compare these factors before and after trainees achieve technical competence.

Methods: Design of this study was a cross-sectional studies of two time series design for one year at a single center. From March 2011 to February 2012, a total 2,050 subjects who underwent colonoscopy by four first-year gastrointestinal fellows were enrolled at Christian hospital, Wonju, Republic of Korea. Four gastrointestinal fellows have filled out the colonoscopic documentation. Main outcome measurement was predictive factors affecting cecal intubation failure and learning curves.

Results: Colonoscopy was successfully completed to the cecum in 1,720 patients (83.9\%). Success rates gradually increased as trainees performed more colonoscopies: the rate of SCI was $62 \%$ in the first 50 cases, and grew to $93 \%$ by the 250th case. Logistic regression analysis of factors affecting cecal intubation failure showed that female gender, low BMI (BMI $<18.5 \mathrm{~kg} / \mathrm{m} 2)$, poor bowel preparation, and past history of stomach surgery were more often associated with cecal intubation failure, particularly before the trainees achieved technical competence.

Conclusion: Several patient characteristics were identified that may predict difficulty of cecal intubation in colonoscopy trainees. Particularly, low BMI, inadequate bowel cleansing, and previous stomach operation were predictors of cecal intubation failure before the trainees have reached technical competency. The results could be informative so that trainees enhance the success rate regarding better colonoscopy training programs.
\end{abstract}

\section{Background}

Colonoscopy is a widely used procedure for the screening and surveillance of colorectal cancer. Colonoscopy and polypectomy have effectively reduced the incidence of colorectal cancer $[1,2]$. However, providing a safe and optimal colonoscopy is not easy even after considerable training and experience. Successful cecal intubation (SCI) is a primary quality indicator in colonoscopies. $\mathrm{SCI}$ is also an important marker in a colonoscopy trainee's progress. However, cecal intubation rates in the early learning phase have been reported as only 56-75\% [3-6]. Furthermore, delayed or failed cecal intubation can cause unfavorable

\footnotetext{
* Correspondence: hyskim@yonsei.ac.kr

'Department of Internal Medicine, Yonsei University Wonju College of

Medicine, Wonju, Republic of Korea

${ }^{2}$ Division of Gastroenterology \& Hepatology, Department of Internal

Medicine and Institute of Lifelong Health, Yonsei University Wonju College of Medicine, 162, Ilsan-dong, Wonju, Gangwon-do 220-701, Korea
}

events, such as patient discomfort, barotrauma and consecutive cecal re-insertion failure, especially if being performed by a trainee. Previous studies have reported the various patient-related factors associated with colonoscopy outcomes. These factors included the age, gender, high or low BMI, bowel preparation, and a history of abdominal surgery and/or peritonitis [7-10]. However, there are limited data about how these factors affect cecal intubation in colonoscopy trainees $[4,5]$. The goal of this study was to determine the factors that affect the failure of cecal intubation and to compare these factors before and after trainees achieve competence.

\section{Methods}

From March 2011 to February 2012, a single-center cross-sectional observational study was conducted at Wonju Christian hospital for 1 year. Colonoscopic indications included screening/surveillance, evaluation of

\section{Biomed Central}


symptoms such as abdominal pain, discomfort, melena. A total of 2,050 colonoscopies were done by four firstyear gastrointestinal (GI) fellows. Patients were excluded for the following reasons: (1) urgent colonoscopy, (2) intended therapeutic colonoscopy, (3) colon obstruction, (4) history of colorectal surgery, and (5) patient refusal. Informed consent was obtained before the procedure. The Ethical Committee at Wonju Christian Hospital approved this study protocol.

\section{Participants}

The participants included four first-year GI fellows involved in colonoscopy training for 12 months. None of the participating fellows had any colonoscopy experience before beginning their fellowship. Each of the four GI fellows completed 450-600 colonoscopies throughout the course of 1 year. Oral consent for their participation in the study was obtained.

\section{Colonoscopy}

All colonoscopies ware performed with an Olympus CF-260 video colonoscope (Olympus Optical Co, Ltd, Tokyo, Japan) after preparatory bowel cleansing with a 4-L of polyethylene glycol solution. We used the following bowel preparation scale: excellent (no or minimal solid stool and only small amount of clear fluid), good (no or minimal solid stool with large amount of clear fluid), fair (collections of semisolid debris that are cleared with difficulty) and poor (solid or semisolid debris that cannot be effectively cleared) [11]. Midazolam and propofol were given to the patient on demand as a bolus. $50 \mathrm{mg}$ of IV pethidine was given prior to the procedure, unless contraindicated for that particular patient. We have both a training protocol for colonoscopy trainees based on quality measurement for colonoscopy and a skill assessment form [12]. Initially, the trainees were instructed to understand indications, processes of procedures, medication of sedation, and descriptions of findings followed by observation of colonoscopy performed by the faculty member or professor for 2 weeks. Then, they were granted to operate the sigmoidoscope for 2 weeks followed by performing colonoscopy only during the withdrawal under the supervisor's watching in 20-30 cases. After this level of training for 2 months, they started the insertion of the scope under supervisor's attendance. All of trainees filled the self assessment form which included patients demographics, histories, indications, and variables related to procedure, complication and pathologic report. Every 50 procedures, all trainees took feed-backs from the senior doctor for their technical assessment; cecal intubation rate, times for cecal intubation and withdrawal, complication rate, and cognitive skills including polyp and adenoma detection rate [13]. Trainees were given 15 minutes to intubate the cecum without the assistance of senior fellows or staff. SCI was defined as the successful photo-documentation of the cecal strap folds and IC valve within 15 minutes, without any assistance. We defined 15 minutes as a competent cecal intubation time based on the previous study results [14]. The nurses recorded cecal intubation time and withdrawal time in order to calculate averages. Withdrawal time did not include time spent on procedures such as biopsy and polypectomy.

\section{Data collection}

We recorded various parameters potentially related to SCI such as age, sex, body mass index (BMI), bowel preparation scale, indication for colonoscopy, and prior surgical history. Three levels of BMI were defined as follows: thin (BMI < 18.5), normal (BMI: 18.5-24.9), over weight (BMI $\geq 25$ ) [15]. These parameters have been analyzed at the first session of 250 cases when trainees did not reach the steady learning curve. Then next analysis was performed when they reached the steady learning curve with achieving over $90 \%$ SCI at next $250-300$ cases respectively.

\section{Statistical analysis}

All analysis was conducted using SPSS, version 18.0 (SPSS Inc., Chicago, IL, USA). The learning curve of trainees was calculated in consecutive blocks of 50 procedures. An independent t-test was used to compare the mean value. The Chi-square test and multivariate logistic regression analysis was used to evaluate the effect of each factor on the failure of cecal intubation. A p-value $<0.05$ was considered significant.

\section{Results}

A total of 2,050 colonoscopies performed from March 2010 to February 2012 were included in the study. Mean patient age was $57.3 \pm 13.8$ years, and the mean BMI was $23.9 \pm$ 3.6. The patients included 1,236 men (60.3\%) and 814 women $(39.7 \%)$. Of the patients, $82.0 \%$ had no history of abdominal surgery, $5.4 \%$ had a history gynecologic surgery, and $3.1 \%$ had a previous stomach operation (subtotal gastrectomy, total gastrectomy, or partial gastrectomy). Excellence in bowel preparation was achieved in $17.7 \%$ of the patients, and good, fair and poor were $70.3 \%, 9.9 \%$ and $2.1 \%$, respectively. The most common indication for colonoscopy was screening and surveillance (57.8\%). During the procedure, there was 1 case of colon perforation and 5 cases of post-polyepectomy or biopsy-induced bleeding which required hospitalization. Baseline characteristics of patients and procedures before and after colonoscopic competency are summarized in Table 1 . In the analyses comparing pre- and post-competency period, also, there were no significant differences in the patients factors responsible for failure except gender; Women were more prevalent in pre-competency period. 
Table 1 Baseline characteristics of patients and procedures before and after colonoscopic competency

\begin{tabular}{|c|c|c|c|c|}
\hline & Total & Pre-competency & Post-competency & $P$ value \\
\hline Age (\%) & & & & 0.447 \\
\hline$<60$ & 55.9 & 55.0 & 56.7 & \\
\hline$>60$ & 44.1 & 45.0 & 43.3 & \\
\hline Sex (\%) & & & & 0.004 \\
\hline Male & 60.3 & 57.1 & 63.3 & \\
\hline Female & 39.7 & 42.9 & 36.7 & \\
\hline BMI (\%) & & & & 0.105 \\
\hline $18.5-25$ & 52.1 & 61.7 & 59.1 & \\
\hline$>25$ & 29.0 & 31.4 & 35.5 & \\
\hline$<18.5$ & 18.5 & 6.9 & 5.4 & \\
\hline Bowel preparation (\%) & & & & 0.154 \\
\hline Excellent/Good & 88.0 & 86.5 & 91.0 & \\
\hline Fair/Poor & 12.0 & 13.5 & 9.0 & \\
\hline \multicolumn{5}{|l|}{ Previous surgery (\%) } \\
\hline \multirow[t]{2}{*}{ Stomach } & 3.1 & 3.5 & 2.7 & 0.307 \\
\hline & 96.9 & 96.5 & 97.3 & \\
\hline \multirow[t]{2}{*}{ Gynecologic } & 5.4 & 5.7 & 5.0 & 0.557 \\
\hline & 94.6 & 94.3 & 95.0 & \\
\hline Hepatobiliary Yes & 1.1 & 1.1 & 1.0 & 0.908 \\
\hline No & 98.9 & 98.9 & 99.0 & \\
\hline \multicolumn{5}{|c|}{ Indication for colonoscopy (\%) } \\
\hline Screening/Surveillance & 57.8 & 56.9 & 58.8 & 0.470 \\
\hline Symptomatic & 34.4 & 34.9 & 33.2 & 0.427 \\
\hline Diagnostic & 3.4 & 3.5 & 3.3 & 0.835 \\
\hline
\end{tabular}

\section{Learning curve}

The overall success rate for successful cecal intubation in less than 15 minutes was $83.9 \%(1,720 / 2,050)$. The trainees' learning curve was calculated in consecutive blocks of 50 colonoscopies. Success rates gradually increased as trainees acquired more colonoscopy experience, from $62 \%$ in the first 50 cases to $93 \%$ in the $250-300$ th cases $(62.0 \%$, $68.5 \%, 76 \%, 80.5 \%, 85.5 \%, 92.5 \%$ and $93.4 \%$, respectively for every 50 consecutive blocks (Figure 1). After 250 colonoscopies, the success rate in reaching the cecum was consistently above $90 \%$ in all four fellows.

\section{Cecal intubation time and withdrawal time}

The mean cecal intubation time was $9.8 \pm 6.8$ minutes, and the mean withdrawal time was $11.1 \pm 4.9$ minutes. The mean cecal intubation time was inversely proportional to the number of colonoscopies the trainee had performed (14.4 min, $12.0 \mathrm{~min}, 8.7 \mathrm{~min}, 6.6 \mathrm{~min}$ for 50 , 150, 250, 500 cases respectively (Figure 2). The mean withdrawal time also decreased as trainee experience increased, but plateaued around 10 minutes after 150 procedures (14.3 $\mathrm{min}, 11.1 \mathrm{~min}, 10.5 \mathrm{~min}, 9.6 \mathrm{~min}$ for $50,150,250,500$ cases respectively (Figure 3 ).

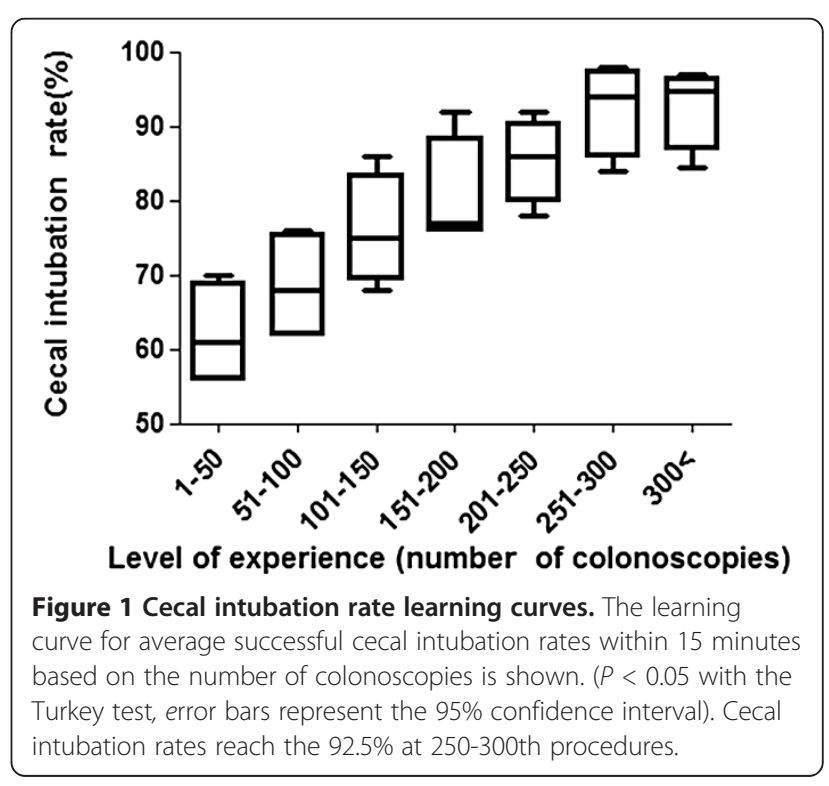




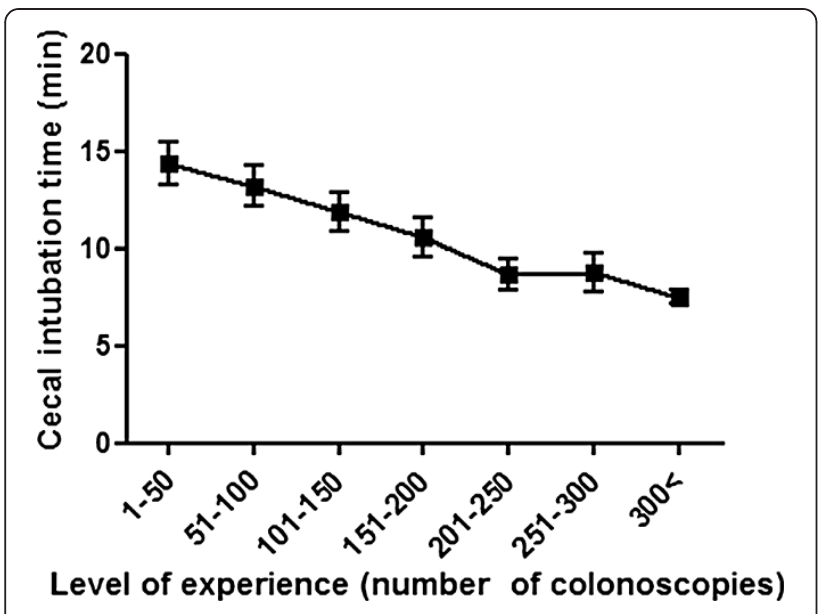

Figure 2 Cecal intubation time learning curves. The learning curve for average cecal intubation times is shown. (Error bars represent the 95\% confidence interval). A significant inverse correlation between cecal intubation times and level of experience is shown.

\section{Unsuccessful cecal intubation}

Of the 2,050 colonoscopies, cecal intubation failed in 330 cases (16.1\%). The most common anatomic site reached by the trainees during failed colonoscopies was the hepatic flexure (31.5\%), followed by the transverse colon (17.9\%), and sigmoid-descending junction (13.9\%). In failed cases, reinsertion by the senior fellows or staff achieved SCI $97.3 \%$ of the time.

The logistic regression results are shown in Table 2. Trainees were 1.3 times as likely to fail to intubate the cecum in older patients (> 60). However, this finding was not statistically significant $(\mathrm{p}=0.064)$. Trainees were 1.5 times more likely to fail to intubate the cecum

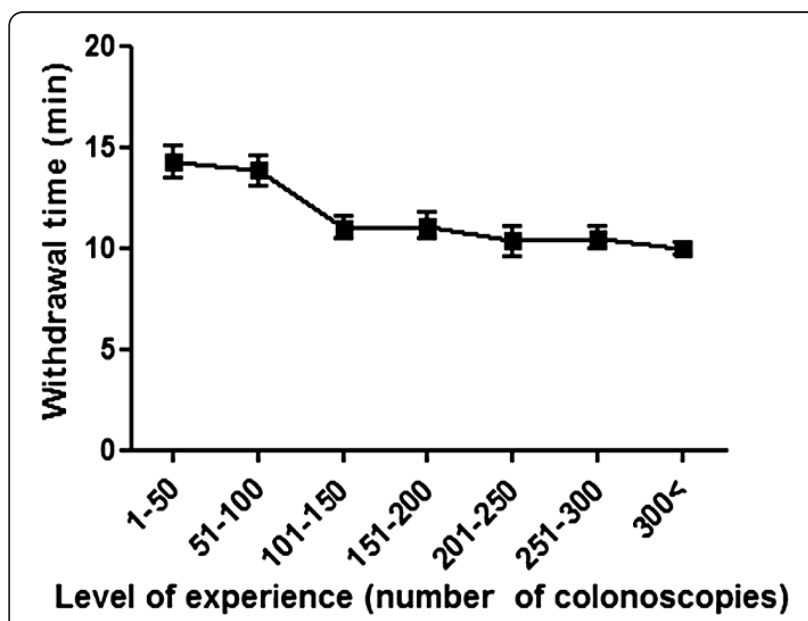

Figure 3 Withdrawal time learning curves. The learning curve for average withdrawal times is shown. (Error bars represent the 95\% confidence interval). Withdrawal times decrease with the level of experience, but steady around 10 minutes after 150 procedures. in females than in males. Patients with low BMI $(<18.5)$ were 1.8 times more difficult to achieve SCI in than normal BMI (18.5-24.9). Additionally, patients with inadequate bowel preparation or with a history of stomach operation were more difficult for the trainees to achieve SCI in than subjects with good bowel preparation and no history of stomach operation by 2.1 times and 3.3 times, respectively. The Hosmer-Lemeshow goodnessof-fit test indicated that this model was a good fit for the data $(\mathrm{p}=0.855)$. A significant difference in the cecal intubation rate was not found according to the history of gynecologic and hepatobiliary surgery, indication for colonoscopy, or ASA status.

After 250 procedures, the success rate for cecal intubation stabilized at over $90 \%$. Because of this, we compared the predictive factors before and after 250 procedures. Interestingly, by logistic regression analysis, age and gender did not show any difference in cecal intubation rate in the trainees before achieving competence. Only low BMI, inadequate bowel preparation, and previous stomach surgery were associated with cecal intubation failure: 1.9 times, 2.0 times, and 2.2 times more likely, respectively. After trainees achieved competency, poor bowel preparation and history of stomach surgery were associated with the failure of cecal intubation. The results are summarized in Table 3.

\section{Discussion}

To perform a colonoscopy safely and effectively, the ability to reach and examine the cecum is necessary. Thus, successful cecal intubation is an obligatory measure of competence. Previous reports indicate that experienced endoscopists consistently achieve cecal intubation in more than $90 \%$ of their procedures, and rates above $90 \%$ have generally been the benchmark for competency $[16,17]$. However, cecal intubation rates in the early phase of training are nowhere near this benchmark [3-6]. Our study considered cecal intubation successful only if cecal landmarks (cecal strap fold and ileocecal valve) were clearly photo-documented within 15 minutes without assistance. The success rate in trainees' fist 50 procedures was only $62 \%$, which is in agreement with previous results $[4,5]$. The success rate for cecal intubation was $62.0 \%, 68.5 \%$, $76.0 \%, 80.5 \%, 85.5 \%, 92.5 \%$ and $93.4 \%$ for every 50 consecutive blocks, respectively. After 250 procedures, the success rate steadily increased above $90 \%$. Thus, in our study we assessed the colonoscopic competence based on the first 250 procedures, and attempted to discern predictors of SCI before and after trainees achieved competency.

Prolonged cecal intubation can cause patients discomfort, barotraumas, or consecutive reinsertion failure, even at the hand of an experienced colonoscopist. Thus, it is important to determine and to avoid factors that affect cecal intubation failure by trainees, especially in 
Table 2 Factors affecting the cecal intubation failure by logistic regression analysis

\begin{tabular}{|c|c|c|c|c|}
\hline & Failure rate (\%) & Odds ratio & $95 \% \mathrm{Cl}$ & $P$ value \\
\hline \multicolumn{5}{|l|}{ Age } \\
\hline$<60$ & 13.6 & 1 & & \\
\hline$>60$ & 19.3 & 1.287 & $0.985-1.681$ & 0.064 \\
\hline \multicolumn{5}{|l|}{ Sex } \\
\hline Male & 13.7 & 1 & & \\
\hline Female & 19.8 & 1.518 & $1.164-1.980$ & 0.002 \\
\hline \multicolumn{5}{|l|}{ BMl } \\
\hline $18.5-25$ & 15.7 & 1 & & \\
\hline$>25$ & 12.6 & 0.808 & $0.600-1.088$ & 0.159 \\
\hline$<18.5$ & 26.9 & 1.771 & $1.108-2.830$ & 0.017 \\
\hline \multicolumn{5}{|l|}{ Bowel preparation } \\
\hline Excellent/Good & 14.8 & 1 & & \\
\hline Fair/Poor & 25.6 & 2.072 & $1.464-2.933$ & 0.000 \\
\hline \multicolumn{5}{|l|}{ Previous surgery } \\
\hline \multirow[t]{2}{*}{ Stomach } & 15.3 & 1 & & \\
\hline & 41.3 & 3.323 & $1.892-5.835$ & 0.000 \\
\hline \multirow{2}{*}{$\begin{aligned} & \text { Gynecologic } \text { No } \\
& \text { Yes }\end{aligned}$} & 15.7 & 1 & & \\
\hline & 23.6 & 1.422 & $0.820-2.412$ & 0.201 \\
\hline
\end{tabular}

Table 3 Factors affecting the cecal intubation failure before and after colonoscopic competency by logistic regression analysis

\begin{tabular}{|c|c|c|c|c|c|c|c|c|}
\hline & \multicolumn{4}{|l|}{ Pre-competency } & \multicolumn{4}{|l|}{ Post-competency } \\
\hline & Failure rate (\%) & Odds ratio & $95 \% \mathrm{Cl}$ & $p$-value & Failure rate (\%) & Odds ratio & $95 \% \mathrm{Cl}$ & $p$-value \\
\hline \multicolumn{9}{|l|}{ Age } \\
\hline$<60$ & 22.0 & 1 & & & 5.7 & 1 & & \\
\hline$>60$ & 29.8 & 1.210 & $0.872-1.678$ & 0.253 & 9.0 & 1.310 & $0.773-2.219$ & 0.316 \\
\hline \multicolumn{9}{|l|}{ Sex } \\
\hline Male & 22.4 & 1 & & & 6.2 & 1 & & \\
\hline Female & 29.6 & 1.325 & $0.953-1.842$ & 0.095 & 8.8 & 1.668 & $0.973-2.859$ & 0.063 \\
\hline \multicolumn{9}{|l|}{ BMI } \\
\hline $18.5-24.99$ & 25.8 & 1 & & & 6.6 & 1 & & \\
\hline$\geq 25$ & 21.3 & 0.783 & $0.545-1.124$ & 0.185 & 5.9 & 1.008 & $0.565-1.798$ & 0.978 \\
\hline$<18.5$ & 40.4 & 1.902 & $0.986-3.189$ & 0.028 & 11.8 & 1.370 & $0.521-3.607$ & 0.524 \\
\hline \multicolumn{9}{|l|}{ Bowel preparation } \\
\hline Excellent/Good & 23.5 & 1 & & & 6.5 & 1 & & \\
\hline Fair/ Poor & 39.7 & 2.037 & $1.312-3.162$ & 0.002 & 12.0 & 2.370 & $1.240-4.529$ & 0.008 \\
\hline \multicolumn{9}{|l|}{ Previous surgery } \\
\hline \multirow[t]{2}{*}{ Stomach } & 24.6 & 1 & & & 6.6 & 1 & & \\
\hline & 51.4 & 2.189 & $1.044-4.587$ & 0.002 & 28.6 & 6.643 & $2.651-16.650$ & 0.000 \\
\hline \multirow{2}{*}{$\begin{aligned} & \text { Gynecologic } \text { No } \\
& \text { Yes }\end{aligned}$} & 24.8 & 1 & & & 7.0 & 1 & & \\
\hline & 36.8 & 1.609 & $0.838-3.088$ & 0.153 & 9.4 & 0.931 & $0.272-3.183$ & 0.909 \\
\hline
\end{tabular}


the early learning phase. The results in this study showed that predictive factors for cecal intubation failure by trainees were female gender, low BMI, poor bowel preparation, and prior stomach operation. Women had a lower cecal intubation rate than men, which was also reported in previous studies $[4,5]$. This could be explained by the fact that the colon is typically longer in women, women may have more unknown anatomic variations, or because of complications as a result of previous gynecologic surgeries $[18,19]$. However, gynecologic surgery did not show any significance for cecal intubation failure in this study.

In patients with low BMI $(<18.5)$, trainees were more likely to fail to reach the cecum within 15 minutes, which is in agreement with results from in a previous study [4]. Patients with a low BMI had a sigmoid colon that was more redundant or difficult [10]. One possible explanation for this finding may be lower amounts of fat, which provides resistance to the colonoscope and thus helps to decrease sigmoid mobility.

There have been no previous reports regarding the relationship between colonoscopy and stomach surgery. In subtotal or total gastrectomy, the surgeon dissects the greater omentum, which is attached to stomach and transverse colon. This may result in the formation of adhesions and anatomic variations around the transverse colon [20]. A significant difference in the cecal intubation failure rate was associated with patient history of stomach operation in the study. In contrast with gynecologic or hepatobiliary operation, trainees had only a $58.7 \%$ success rate for cecal intubation if the patient had a history of stomach operation. In the cases of stomach operation, the most common anatomic site reached by the trainees during failed colonoscopies was the transverse colon (38.5\%) and sigmoid-descending junction (34.6\%). In addition, most trainees in the early phase of colonoscopy insert the colonoscope by push-type, and try to reduce the loop after reaching the transverse colon. However, if there are adhesions around the transverse colon, there may be insufficient shortening of the colon, thus creating difficulties in the straightening of the scope.

In this study, 250 procedures were required for trainees to achieve competence in colonoscopy within 15 minutes. These results are very similar to those of previous studies $[6,14]$. This supports a previous study that an average of 275 procedures performed within 16 minutes are needed for the average GI trainee to meet minimal competence criteria. Two prior studies showed that the success rate significantly improved and trainees reached the requisite standard competence (>90\%) after 150-200 cases, which is even less than the 250 cases determined in our study $[4,5]$. The reason for this discrepancy is probably because our study gave trainees less time to intubate the cecum. The published benchmarks for cecal insertion time for minimal competency range from 15 to 20 minutes [6,14]. Even in the first 50 cases, the mean time to reach the cecum was less than 15 minutes and decreased as trainees acquired more experience.

There are several limitations to our study. First, it may be difficult to generalize these predictive factors for cecal intubation failure since this is a single-center study. Also, we considered only the patient's factors, and did not look at factors such as assistants' experience, or the fatigue of the colonoscopist. In addition, it is clear that fellows acquired the skill at different rates, but we did not consider the differences between fellows by this measure. The number of trainees participated and the number of subjects with previous stomach surgery included in this study was relatively small. Furthermore, the study patients may pose a selected group because the majority of procedures were indicated by screening or surveillance. For ethical reasons, however, we included only patients with good general condition (ASA class 1 or 2 ) in order to avoid potential risks of procedure related complications from the trainee's incomplete skill acquaintance. Finally, colonoscopy method using assistant device or technique such as cap or water immersion was not taught to the trainees when they failed the cecal intubation. Several kinds of technical assistances may be helpful for trainees to perform difficult colonoscopy. In particular, there are emerging data that warm water immersion (WWI) technique enhanced cecal intubation rate and willingness to undergo a repeat colonoscopy. Also, WWI can not only reduce the number of patients requiring on-demand sedation but also improve the overall patients tolerance of colonoscopy. Therefore, it would be very interesting whether this technique facilitates the learning curve in the trainee [21,22].

\section{Conclusion}

Several patient characteristics were identified that may predict difficulty of cecal intubation in colonoscopy trainees. The results could be informative so that trainees enhance the success rate regarding better colonoscopy training programs, as well as that they ensure patient safety in the early phase of the learning curve. Particularly, before trainees acquire competence, it might be advised for trainees to avoid performing this procedure on patients who are of low BMI, have inadequate bowel cleansing, or a history of stomach surgery.

\section{Competing interests}

The authors declare that they have no competing interests.

\section{Authors' contributions}

This study was supported by a grant of the Korean Health Technology R\&D Project, Ministry for Health, Welfare \& Family Affairs (A102065-23) and a grant from the National R\&D Program for Cancer Control, Ministry of Health \& Welfare, Republic of Korea (1220230). All authors read and approved the final manuscript. 


\section{References}

1. Citarda F, Tomaselli G, Capocaccia R, et al: Efficacy in standard clinical practice of colonoscopic polypectomy in reducing colorectal cancer incidence. Gut 2001, 48:812-815.

2. Zauber AG, Winawer SJ, O'Brien MJ, et al: Colonoscopic polypectomy and long-term prevention of colorectal-cancer deaths. N Engl J Med 2012, 366:687-696.

3. Dafnis G, Granath F, Pahlman L, et al: The impact of endoscopists' experience and learning curves and interendoscopist variation on colonoscopy completion rates. Endoscopy 2001, 33:511-517.

4. Lee SH, Chung IK, Kim SJ, et al: An adequate level of training for technical competence in screening and diagnostic colonoscopy: a prospective multicenter evaluation of the learning curve. Gastrointest Endosc 2008, 67:683-689.

5. Chung Jl, Kim N, Um MS, et al: Learning curves for colonoscopy: a prospective evaluation of gastroenterology fellows at a single center. Gut Liver 2010, 4:31-35.

6. Spier BJ, Benson M, Pfau PR, et al: Colonoscopy training in gastroenterology fellowships: determining competence. Gastrointest Endosc 2010, 71:319-324.

7. Oh SY, Sohn Cl, Sung IK, et al: Factors affecting the technical difficulty of colonoscopy. Hepatogastroenterology 2007, 54:1403-1406.

8. Shah HA, Paszat LF, Saskin R, et al: Factors associated with incomplete colonoscopy: a population-based study. Gastroenterology 2007, 132:2297-2303.

9. Bernstein C, Thorn M, Monsees $\mathrm{K}$, et al: A prospective study of factors that determine cecal intubation time at colonoscopy. Gastrointest Endosc 2005, 61:72-75.

10. Anderson JC, Messina CR, Cohn W, et al: Factors predictive of difficult colonoscopy. Gastrointest Endosc 2001, 54:558-562.

11. DiPalma JA, Wolff BG, Meagher A, et al: Comparison of reduced volume versus four liters sulfate-free electrolyte lavage solutions for colonoscopy colon cleansing. Am J Gastroenterol 2003, 98:2187-2191.

12. Rex DK, Petrini JL, Baron TH, Chak A, Cohen J, Deal SE, et al: Quality indicators for colonoscopy. Am J Gastroenterol 2006, 101(4):873-885.

13. Sedlack RE: The mayo colonoscopy skills assessment tool: validation of a unique instrument to assess colonoscopy skills in trainees. Gastrointest Endosc 2010, 72(6):1125-1133. 1133.e1-3.

14. Sedlack RE: Training to competency in colonoscopy: assessing and defining competency standards. Gastrointest Endosc 2011, 74:355-366. e1-2.

15. WHO expert consultation: Appropriate body-mass index for asian populations and its implications for policy and intervention strategies. Lancet 2004, 363:157-163.

16. Chak A, Cooper GS, Blades EW, et al: Prospective assessment of colonoscopic intubation skills in trainees. Gastrointest Endosc 1996, 44:54-57.

17. Lieberman DA, Faigel DO, Logan JR, et al: Assessment of the quality of colonoscopy reports: results from a multicenter consortium. Gastrointest Endosc 2009, 69:645-653.

18. Waye JD, Bashkoff E: Total colonoscopy: is it always possible? Gastrointest Endosc 1991, 37:152-154.

19. Church JM: Complete colonoscopy: how often? and if not, why not? Am J Gastroenterol 1994, 89:556-560.

20. Varela JE, Hiyashi M, Nguyen T, et al: Comparison of laparoscopic and open gastrectomy for gastric cancer. Am J Surg 2006, 192:837-842.

21. Leung JW, Mann SK, Siao-Salera R, Ransibrahmanakul K, Lim B, Cabrera H, et al: A randomized, controlled comparison of warm water infusion in lieu of air insufflation versus air insufflation for aiding colonoscopy insertion in sedated patients undergoing colorectal cancer screening and surveillance. Gastrointest Endosc 2009, 70(3):505-510.

22. Leung FW, Harker JO, Jackson G, Okamoto KE, Behbahani OM, Jamgotchian NJ, et al: A proof-of-principle, prospective, randomized, controlled trial demonstrating improved outcomes in scheduled unsedated colonoscopy by the water method. Gastrointest Endosc 2010, 72(4):693-700.

\section{Submit your next manuscript to BioMed Central and take full advantage of:}

- Convenient online submission

- Thorough peer review

- No space constraints or color figure charges

- Immediate publication on acceptance

- Inclusion in PubMed, CAS, Scopus and Google Scholar

- Research which is freely available for redistribution 